\title{
A Multiple Attribute Decision-Making Method Based on Exponential Fuzzy Numbers
}

\author{
Sha Fu ${ }^{1,2, *}$ and Guo-bing Fan ${ }^{1}$ \\ 1 Department of Information Management, Hunan University of Finance and Economics, \\ Changsha 410205, China \\ 2 DeVry University, Fremont, CA 94555, USA \\ * Correspondence: fusha15@163.com; Tel.: +13-875-982-792 \\ Academic Editor: Mehmet Ilgin \\ Received: 23 April 2016; Accepted: 17 May 2016; Published: 21 May 2016
}

\begin{abstract}
Regarding the multi-attribute decision-making problem where both the attribute value and attribute weight of a scheme are exponential fuzzy numbers, this paper proposes a fuzzy multiple attribute decision-making method based on exponential fuzzy numbers. The expected value and variance of the exponential fuzzy number were calculated according to the definitions of expectation and variance in probability theory. By comprehensively considering factors such as expected value, variance, and attitude preference of the decision-maker, this paper gave the score function of the exponential fuzzy number, based on which the accurate attribute weight can be determined. Subsequently, based on the distance measure between exponential fuzzy numbers, the distance was calculated between each scheme and the positive/negative ideal scheme, respectively, and the relative closeness of each scheme was solved; based on this, sorting and prioritizing were conducted. Finally, the feasibility and effectiveness of the proposed method were verified through case analysis.
\end{abstract}

Keywords: exponential fuzzy number; fuzzy multiple attribute decision making; expected value; weighted distance; relative closeness

\section{Introduction}

The multi-attribute decision-making (MADM) problem is one of the key sectors in modern decision science, the theory and method of which have been widely applied in the fields of engineering design, social life, investment decision-making and project evaluation. The multi-attribute decision-making problem is of profound theoretical significance and has a wide practical application background in various industries; therefore, research on multi-attribute decision-making problems has always been a key subject for people. In real life, the uncertainty of decision information is regularly caused due to complex object environments and the fuzziness of human thinking. In solving such a class of problems, fuzzy numbers such as interval numbers, triangular fuzzy numbers, and trapezoidal fuzzy numbers are usually adopted to express such uncertainty of decision-making information.

In recent years, research on fuzzy numbers has attracted attention from scholars and experts, and has been widely used in the field of multi-attribute decision-making problems [1-13]. For example, Didier Dubois et al. [2] provided a justification of symmetric triangular fuzzy numbers in the spirit of such inequalities. It shows that the cuts of such a triangular fuzzy number contain the "confidence intervals" of any symmetric probability distribution with the same mode and support. Lakshmana et al. [3] proposed and discussed a new method for the sequencing of a interval-valued intuitionistic fuzzy set, and stated this method by the way of calculation example analysis, and made a comparison with other methods as well. Park et al. [4] extended the TOPSIS (Technique for Order Preference by Similarity to Ideal Solution) method in order to solve problems of multi-attribute 
group decision-making (MAGDM) in interval-valued intuitionistic fuzzy circumstances, in which all preferential information provided by decision-makers would be indicated by an interval-valued intuitionistic fuzzy decision matrix. Xu [5] proposed an ideal method to solve the problem with interval-valued intuitionistic fuzzy MADM with the attribute weight not completely known or completely unknown. References [6,7] define the desired value and expected scores of intuitionistic trapezoidal fuzzy numbers (ITFN) and the orderly weighted integration operator, and establish a multi-attribute group decision-making model based on ITFN. Wang Jian-qiang and Wei Gui-wu $[8,9]$ established an objective programming model based on the distance measure and difference maximum, respectively, and proposed a multiple attribute decision-making method where the attribute weight information is incomplete and the attribute value is an interval-valued intuitionistic fuzzy number. Wan [10], in a multi-attribute decision-making problem where both the decision-maker's preferential information and attribute value are interval numbers, conducted specific research on the attitude index method of the interval multiple attribute decision-making problem. Chen and Yang [11], regarding the multi-attribute group decision-making problem where the assessment information of the scheme attribute and attribute weight are in fuzzy language form, proposed a method to solve a fuzzy multi-attribute group decision-making problem based on the concept and property of triangular fuzzy numbers. Wang and Zhang [12] conducted research on multiple-criteria decision-making problems where the criterion value is an intuitionistic trapezoidal fuzzy number and the information is incomplete, respectively, and proposed a corresponding decision-making method. Chen et al. [13] established a layered multi-objective linear programming model based on trapezoidal fuzzy numbers in the hope of solving multi-attribute decision-making problems in practical life, and then solved this model using the layered sequence optimization method and compared it with the Nezhad model for further analysis. Chen [14], by combining the theory of the fuzzy ideal point, proposed a TOPSIS multi-attribute decision-making method through defining the expected value of the exponential fuzzy number and the distance measure between exponential fuzzy numbers. Xu and Wang [15] presented a comprehensive review on relative developments of multi-granularity linguistic term sets using several perspectives. Most of the above research on fuzzy multiple attribute decision-making problems mainly involves interval numbers, triangular fuzzy numbers and trapezoidal fuzzy numbers, while there is less research on fuzzy multiple attribute decision-making problems based on exponential fuzzy numbers. This paper, regarding the current research situation, proposes a corresponding decision-making method to meet the requirements of such decision-making problems.

\section{Preliminaries}

Definition 1. If the membership function of $\tilde{a}$ can be expressed as:

$$
u_{\widetilde{a}}(x)=\left\{\begin{array}{l}
\exp \left[-\left(\frac{x-c}{\sigma}\right)^{2}\right], x \leqslant c \\
\exp \left[-\left(\frac{x-c}{\tau}\right)^{2}\right], x>c
\end{array}\right.
$$

Therefore, $\tilde{a}=(c, \sigma, \tau)$ is an exponential fuzzy number. Suppose $R$ is donated as a real number set, then all exponential fuzzy numbers in $R$ are denoted as $F E(R)$ [14].

Definition 2. Suppose $\tilde{a}_{1}=\left(c_{1}, \sigma_{1}, \tau_{1}\right), \widetilde{a}_{2}=\left(c_{2}, \sigma_{2}, \tau_{2}\right) \in F E(R)$ is an exponential fuzzy number; thus,

1. $\tilde{a}_{1}+\tilde{a}_{2}=\left(c_{1}+c_{2}, \sigma_{1}+\sigma_{2}, \tau_{1}+\tau_{2}\right)$

2. $k \tilde{a}_{1}=\left(k c_{1}, k \sigma_{1}, k \tau_{1}\right), k>0$

Definition 3. Suppose $\tilde{a}=(c, \sigma, \tau) \in F E(R)$; the expected value of $\tilde{a}$ is:

$$
E(\widetilde{a})=\int_{-\infty}^{+\infty} x u_{\widetilde{a}}(x) d x
$$

where the expectation reflects the average value of the fuzzy number. 
Definition 4. Suppose $\tilde{a}=(c, \sigma, \tau) \in F E(R)$; the variance of $\tilde{a}$ is:

$$
\operatorname{Var}(\widetilde{a})=\int_{-\infty}^{+\infty}(x-E(\widetilde{a}))^{2} u_{\widetilde{a}}(x) d x
$$

where the variance describes the degree of deviation from the fuzzy number to the average value.

Definition 5. Suppose exponential fuzzy number $\tilde{a}_{1}=\left(c_{1}, \sigma_{1}, \tau_{1}\right), \tilde{a}_{2}=\left(c_{2}, \sigma_{2}, \tau_{2}\right), \lambda \in(0,1)$; therefore, the distance $D\left(\tilde{a}_{1}, \tilde{a}_{2}\right)$ between $\tilde{a}_{1}$ and $\tilde{a}_{2}$ can be expressed as [16]:

$$
\begin{aligned}
& D\left(\tilde{a}_{1}, \tilde{a}_{2}\right)=\sqrt{\int_{0}^{1} d^{2}\left(\left(\tilde{a}_{1}\right)_{\lambda^{\prime}}\left(\tilde{a}_{2}\right)_{\lambda}\right) d_{\lambda}} \\
& =\sqrt{\begin{array}{l}
\left(c_{1}-c_{2}\right)^{2}+\frac{\sqrt{\pi}}{2}\left(c_{1}-c_{2}\right)\left(\left(\tau_{1}-\tau_{2}\right)-\left(\sigma_{1}-\sigma_{2}\right)\right)+ \\
\frac{1}{4}\left(\left(\tau_{1}-\tau_{2}\right)-\left(\sigma_{1}-\sigma_{2}\right)\right)^{2}+\frac{1}{12}\left(\left(\tau_{1}-\tau_{2}\right)+\left(\sigma_{1}-\sigma_{2}\right)\right)^{2}
\end{array}}
\end{aligned}
$$

Definition 6. Suppose exponential fuzzy number $\tilde{a}=(c, \sigma, \tau)$; the score function is:

$$
P(\widetilde{a})=\frac{\phi E(\widetilde{a})}{(1-\phi) \operatorname{Var}(\widetilde{a})}, \phi \in(0,1)
$$

where $E(\tilde{a})$ and $\operatorname{Var}(\tilde{a})$ are the expected value and variance of exponential fuzzy number $\tilde{a}=(c, \sigma, \tau)$, respectively; $P(\widetilde{a})$ reflects the overall effect of the exponential fuzzy number; $\phi$ is the attitude of the decision-maker, which is determined by the degree of the decision-maker's emphasis on the expected value of the decision-making information (average level) and variance (stability level). If $\phi>0.5$, the expected value is attached with more emphasis, while if $\phi<0.5$, the stability level is more emphasized. If $\phi=0.5$, then both factors are equally emphasized [17].

\section{Fuzzy Multi-Attribute Decision-Making Problem Based on an Exponential Fuzzy Number}

\subsection{Problem Description}

Regarding a fuzzy multi-attribute decision-making problem, suppose $A=\left\{A_{1}, A_{2}, \cdots, A_{m}\right\}$ is the scheme set, $C=\left\{C_{1}, C_{2}, \cdots, C_{n}\right\}$ is the attribute set, and $R=\left(\tilde{a}_{i j}\right)_{m \times n}$ is the fuzzy decision-making matrix, where $\tilde{a}_{i j}=\left(c_{i j}, \sigma_{i j}, \tau_{i j}\right)$ represents the exponential fuzzy number attribute value of the $j$ th attribute $C_{j}$ of the $i$ th scheme, $1 \leqslant i \leqslant m, 1 \leqslant j \leqslant n$. In addition, the attribute weight is still given in the form of an exponential fuzzy number, i.e., the attribute weight vector $W=\left(\widetilde{w}_{1}, \widetilde{w}_{2}, \cdots, \widetilde{w}_{n}\right)$, where $\widetilde{w}_{j}=\left(c_{w j}, \sigma_{w j}, \tau_{w j}\right)$ represents the weight of the $j$ th attribute $C_{j}$. Try to sort and prioritize schemes according to the fuzzy decision-making matrix and attribute weight information [18].

\subsection{Decision-Making Steps}

Steps of such a decision-making method are listed below:

Step 1. Construct a fuzzy decision-making matrix $R$ according to the attribute value of each scheme.

Step 2. Standardized processing of the decision-making matrix. Since the attribute values of schemes in the decision-making matrix $R$ have different units of measurement and are subjected to different measurement criteria, in order to achieve unified processing the following equations can be employed for the standardized processing, so as to obtain the standardized decision-making matrix $R^{\prime}=\left(\widetilde{R}_{i j}\right)_{m \times n^{\prime}}$ where $\widetilde{R}_{i j}=\left(\widetilde{c}_{i j}, \widetilde{\sigma}_{i j}, \widetilde{\tau}_{i j}\right)$. In the multi-attribute decision-making problem, the benefit-oriented attribute and cost-oriented attribute are two major attribute types. 
For the benefit-oriented attribute, there is:

$$
\tilde{c}_{i j}=\frac{c_{i j}}{\sum_{i=1}^{m} c_{i j}}, \tilde{\sigma}_{i j}=\frac{\max _{i j}}{\sum_{i=1}^{m} c_{i j}} \sigma_{i j}, \tilde{\tau}_{i j}=\frac{\max c_{i j}}{\sum_{i=1}^{m} c_{i j}} \tau_{i j}
$$

For the cost-oriented attribute, there is:

$$
\widetilde{c}_{i j}=\frac{\frac{1}{c_{i j}}}{\sum_{i=1}^{\frac{1}{m} c_{i j}}}, \tilde{\sigma}_{i j}=\frac{\min \frac{1}{c_{i j}}}{\sum_{i=1}^{m} \frac{1}{c_{i j}}} \sigma_{i j}, \widetilde{\tau}_{i j}=\frac{\min \frac{1}{c_{i j}}}{\sum_{i=1}^{m} \frac{1}{c_{i j}}} \tau_{i j}
$$

Step 3. Determination of positive ideal scheme $A^{+}$and negative ideal scheme $A^{-}$of the fuzzy multi-attribute decision-making problem.

Positive ideal scheme:

$$
A^{+}=\left[A_{1}^{+}, A_{2}^{+}, \cdots, A_{n}^{+}\right], A_{j}^{+}=\left(c_{j}^{+}, \sigma_{j}^{+}, \tau_{j}^{+}\right)=\left(\max _{i} \widetilde{c}_{i j}, \min _{i} \tilde{\sigma}_{i j}, \max _{i} \tilde{\tau}_{i j}\right)
$$

Negative ideal scheme:

$$
A^{-}=\left[A_{1}^{-}, A_{2}^{-}, \cdots, A_{n}^{-}\right], A_{j}^{-}=\left(c_{j}^{-}, \sigma_{j}^{-}, \tau_{j}^{-}\right)=\left(\min _{i} \widetilde{c}_{i j}, \max _{i} \widetilde{\sigma}_{i j}, \min _{i} \tilde{\tau}_{i j}\right)
$$

where $j=1,2, \cdots, n$.

Step 4. Determination of the weights of the attributes. The weight information of the attribute is given in the form of an exponential fuzzy number, i.e., the attribute weight vector.

$$
W=\left(\widetilde{w}_{1}, \widetilde{w}_{2}, \cdots, \widetilde{w}_{n}\right)=\left\{\left(c_{w 1}, \sigma_{w 1}, \tau_{w 1}\right),\left(c_{w 2}, \sigma_{w 2}, \tau_{w 2}\right), \cdots,\left(c_{w n}, \sigma_{w n}, \tau_{w n}\right)\right\}
$$

Calculate the score value of each attribute using Definition 6.

$$
P\left(\widetilde{w}_{j}\right)=\frac{\phi E\left(\widetilde{w}_{j}\right)}{(1-\phi) \operatorname{Var}\left(\widetilde{w}_{j}\right)}
$$

where $\widetilde{w}_{j}$ represents the weight of the $j$ th attribute $C_{j}$, and $\phi$ represents the decision-maker's attitude preference on the expected value of the decision-making information and variance.

Therefore, the accurate weight of each attribute can be obtained as:

$$
w_{j}=\frac{P\left(\widetilde{w}_{j}\right)}{\sum_{j=1}^{n} P\left(\widetilde{w}_{j}\right)}
$$

Step 5. Calculate the weighed distance between each scheme and the positive/negative ideal scheme.

The weighed distance between scheme $A_{i}$ and the positive ideal scheme:

$$
D_{i}^{+}\left(A_{i}, A^{+}\right)=\sum_{j=1}^{n} w_{j} D\left(A_{i j}, A_{i}^{+}\right)
$$

The weighed distance between scheme $A_{i}$ and the negative ideal scheme:

$$
D_{i}^{-}\left(A_{i}, A^{-}\right)=\sum_{j=1}^{n} w_{j} D\left(A_{i j}, A_{i}^{-}\right)
$$


Step 6. Calculate the relative closeness $\varepsilon_{i}$ of each scheme, and sort schemes according to the value of relative closeness; a larger $\varepsilon_{i}$ value represents a more optimal scheme.

$$
\varepsilon_{i}=\frac{D_{i}^{-}\left(A_{i}, A^{-}\right)}{D_{i}^{+}\left(A_{i}, A^{+}\right)+D_{i}^{-}\left(A_{i}, A^{-}\right)}
$$

\section{Example Analysis}

Suppose there is a fuzzy multi-attribute decision-making problem involving five alternatives $A_{i}(i=1,2, \cdots, 5)$ and six attributes $C_{j}(j=1,2, \cdots, 6)$, where all attributes are benefit-oriented and all attribute values are given in the form of an exponential fuzzy number. See Table 1 . Given that the attribute weight vector $W=((0.2,0.02,0.03),(0.1,0.01,0.01),(0.25,0.03,0.02),(0.1,0.01,0.02),(0.2,0.03,0.02)$, $(0.15,0.01,0.03))$, try to sort and prioritize the five schemes using the method proposed in this paper.

(1) Establishment of the fuzzy decision-making matrix, which is shown in Table 1.

(2) Using Equations (4) and (5) to standardize the fuzzy decision-making matrix so that the standardized decision-making matrix is obtained as shown in Table 2.

(3) Determine the positive and negative ideal schemes using Equations (6) and (7).

Positive ideal scheme:

$A^{+}=[(0.2125,0.0043,0.0128),(0.2058,0.0021,0.0082),(0.2052,0.0041,0.0062),(0.2069,0.0041,0.0083)$,

$(0.2113,0.0021,0.0085),(0.2100,0.0021,0.0063)]$

Negative ideal scheme:

$A^{-}=[(0.1902,0.0106,0.0064),(0.1903,0.0062,0.0041),(0.1901,0.0103,0.0021),(0.1918,0.0062,0.0041)$,

$(0.1939,0.0063,0.0021),(0.1883,0.0063,0.0042)]$

(4) Determine the weight of each attribute. First calculate the score value of each attribute using Equation (8) and then calculate the accurate weight of each attribute using Equation (9); the results are show in Table 3.

(5) Calculate the weighted distance between each attribute and the positive/negative ideal schemes according to Equations (10) and (11), and then calculate the relative closeness of each scheme using Equation (12). The results are shown in Tables 4 and 5.

(6) Sort schemes according to the value of $\varepsilon_{i}$, and the sorting result is $A_{1}>A_{2}>A_{5}>A_{3}>A_{4}$; therefore, $A_{1}$ is the optimal scheme. 
Table 1. Fuzzy decision-making matrix.

\begin{tabular}{|c|c|c|c|c|c|c|}
\hline & $C_{1}$ & $C_{2}$ & $C_{3}$ & $C_{4}$ & $C_{5}$ & $C_{6}$ \\
\hline$A_{1}$ & $(0.85,0.05,0.05)$ & $(0.92,0.02,0.03)$ & $(0.94,0.03,0.01)$ & $(0.96,0.03,0.03)$ & $(0.91,0.01,0.01)$ & $(0.97,0.02,0.02)$ \\
\hline$A_{2}$ & $(0.95,0.05,0.05)$ & $(0.89,0.01,0.03)$ & $(0.94,0.02,0.03)$ & $(0.92,0.02,0.03)$ & $(0.97,0.03,0.01)$ & $(0.93,0.03,0.02)$ \\
\hline$A_{3}$ & $(0.91,0.03,0.04)$ & $(0.86,0.02,0.04)$ & $(0.94,0.03,0.03)$ & $(0.94,0.03,0.02)$ & $(0.89,0.03,0.03)$ & $(0.92,0.01,0.02)$ \\
\hline$A_{4}$ & $(0.87,0.02,0.03)$ & $(0.93,0.02,0.02)$ & $(0.88,0.03,0.02)$ & $(0.89,0.03,0.04)$ & $(0.90,0.03,0.04)$ & $(0.93,0.01,0.03)$ \\
\hline$A_{5}$ & $(0.89,0.03,0.06)$ & $(0.92,0.03,0.03)$ & $(0.95,0.05,0.02)$ & $(0.93,0.02,0.02)$ & $(0.92,0.02,0.04)$ & $(0.87,0.02,0.03)$ \\
\hline
\end{tabular}

Table 2. Standardized decision-making matrix.

\begin{tabular}{ccccccc}
\hline & $C_{1}$ & $C_{\mathbf{2}}$ & $C_{\mathbf{3}}$ & $C_{\mathbf{4}}$ & $C_{\mathbf{5}}$ & \\
\hline$A_{1}$ & $(0.1902,0.0106,0.0106)$ & $(0.2035,0.0041,0.0062)$ & $(0.2030,0.0062,0.0021)$ & $(0.2069,0.0062,0.0062)$ & $(0.1983,0.0021,0.0021)$ & $(0.2100,0.0042,0.0042)$ \\
$A_{2}$ & $(0.2125,0.0106,0.0106)$ & $(0.1969,0.0021,0.0062)$ & $(0.1987,0.0041,0.0062)$ & $(0.1983,0.0041,0.0062)$ & $(0.2113,0.0063,0.0021)$ & $(0.2013,0.0063,0.0042)$ \\
$A_{3}$ & $(0.2036,0.0064,0.0085)$ & $(0.1903,0.0041,0.0082)$ & $(0.2030,0.0062,0.0062)$ & $(0.2026,0.0062,0.0041)$ & $(0.1939,0.0063,0.0063)$ & $(0.1991,0.0021,0.0042)$ \\
$A_{4}$ & $(0.1946,0.0043,0.0064)$ & $(0.2058,0.0041,0.0041)$ & $(0.1901,0.0062,0.0041)$ & $(0.1918,0.0062,0.0083)$ & $(0.1961,0.0063,0.0085)$ & $(0.2013,0.0021,0.0063)$ \\
$A_{5}$ & $(0.1991,0.0064,0.0128)$ & $(0.2035,0.0062,0.0062)$ & $(0.2052,0.0103,0.0041)$ & $(0.2004,0.0041,0.0041)$ & $(0.2004,0.0042,0.0085)$ & $(0.1883,0.0042,0.0063)$ \\
\hline
\end{tabular}

Table 3. Expected value, variance, score value and accurate weight of each attribute.

\begin{tabular}{cccccc}
\hline & Fuzzy weight value & Expected value & Variance & Score value & Accurate weight of each attribute \\
\hline$C_{1}$ & $(0.20,0.02,0.03)$ & 0.00911 & 0.00180 & 5.04855 & 0.12385 \\
$C_{2}$ & $(0.10,0.01,0.01)$ & 0.00177 & 0.00017 & 10.12889 & 0.24848 \\
$C_{3}$ & $(0.25,0.03,0.02)$ & 0.01083 & 0.00254 & 4.25836 & 0.10446 \\
$C_{4}$ & $(0.10,0.01,0.02)$ & 0.00281 & 0.00029 & 9.61984 & 0.23599 \\
$C_{5}$ & $(0.20,0.03,0.02)$ & 0.00861 & 0.00161 & 5.33667 & 0.13092 \\
$C_{6}$ & $(0.15,0.01,0.03)$ & 0.00572 & 0.00090 & 6.37157 & 0.15630 \\
\hline
\end{tabular}


Table 4. The weighted distance between each scheme and the positive/negative ideal schemes.

\begin{tabular}{ccc}
\hline & $A^{+}$ & \multicolumn{1}{c}{$A^{-}$} \\
\hline$A_{1}$ & {$[0.0262,0.0041,0.0051,0.0021,0.0161,0.0021]$} & {$[0.0025,0.0151,0.0149,0.0160,0.0064,0.0226]$} \\
$A_{2}$ & {$[0.0044,0.0098,0.0065,0.0096,0.0053,0.0116]$} & {$[0.0243,0.0095,0.0134,0.0084,0.0174,0.0130]$} \\
$A_{3}$ & {$[0.0119,0.0164,0.0032,0.0072,0.0203,0.0118]$} & {$[0.0163,0.0031,0.0167,0.0108,0.0024,0.0128]$} \\
$A_{4}$ & {$[0.0209,0.0031,0.0170,0.0160,0.0172,0.0087]$} & {$[0.0077,0.0164,0.0031,0.0024,0.0055,0.0159]$} \\
$A_{5}$ & {$[0.0144,0.0052,0.0043,0.0084,0.0119,0.0226]$} & {$[0.0139,0.0142,0.0160,0.0096,0.0105,0.0021]$} \\
\hline
\end{tabular}

Table 5. The weighted distance between each scheme and the positive/negative ideal schemes, relative closeness and sorting result.

\begin{tabular}{lcccc}
\hline & $\boldsymbol{D}_{\mathbf{i}}^{+}$ & $\boldsymbol{D}_{\mathbf{i}}^{-}$ & $\boldsymbol{\varepsilon}_{\mathbf{i}}$ & Sorting result \\
\hline$A_{1}$ & 0.0077 & 0.0138 & 0.6403 & 1 \\
$A_{2}$ & 0.0084 & 0.0131 & 0.6080 & 2 \\
$A_{3}$ & 0.0121 & 0.0094 & 0.4377 & 4 \\
$A_{4}$ & 0.0125 & 0.0091 & 0.4214 & 5 \\
$A_{5}$ & 0.0106 & 0.0109 & 0.5070 & 3 \\
\hline
\end{tabular}




\section{Conclusions}

Regarding the fuzzy multi-attribute decision-making problem, this paper proposed a fuzzy multiple attribute decision-making method based on exponential fuzzy numbers. According to the definitions of expectation and variance in probability theory, and by comprehensively considering the attitude preference of the decision-maker, this method can realize the accurate treatment of attribute weight. Subsequently, based on the distance measure between exponential fuzzy numbers, the distance between each scheme and the positive/negative ideal schemes was calculated, respectively, so as to obtain the relative closeness of each scheme. Finally, the feasibility and effectiveness of the proposed method were verified through case analysis. The proposed decision-making method is superior for clear logic, a simple decision-making process and ease of being understood. In addition, such a method has excellent application value and practical decision-making value, providing a scientific and practical decision-making reference for solving fuzzy multi-attribute decision-making problems.

Acknowledgments: This study was supported by the Hunan Province Education and Science Planning Project (No. XJK014BGD005), by the Hunan Province Philosophy and Social Science Foundation (No. 14YBA065). Supported by the construct program of the key discipline in Hunan Province.

Author Contributions: Sha Fu conceived and designed the experiments. Sha Fu and Guo-bing Fan performed the experiments and analyzed the data. Sha Fu wrote the paper.

Conflicts of Interest: The authors declare no conflict of interest.

\section{References}

1. Jahanshahloo, G.R.; Lotfi, F.H.; Izadikhah, M. An algorithmic method to extend TOPSIS for decision-making problems with interval data. Appl. Math. Comput. 2006, 175, 1375-1384. [CrossRef]

2. Dubois, D.; Foulloy, L.; Mauris, G.; Prade, H. Probability-Possibility Transformations, Triangular Fuzzy Sets, and Probabilistic Inequalities. Reliab. Comput. 2004, 10, 273-297. [CrossRef]

3. Lakshmana Gomathi Nayagam, V.; Muralikrishnan, S.; Sivaraman, G. Multi-Criteria decision-making method based on interval-valued intuitionistic fuzzy sets. Expert Syst. Appl. 2011, 38, 1464-1467. [CrossRef]

4. Park, J.H.; Park, I.Y.; Kwun, Y.C.; Tan, X. Extension of the TOPSIS method for decision making problems under interval-valued intuitionistic fuzzy environment. Appli. Math. Model. 2011, 35, 2544-2556. [CrossRef]

5. Xu, Z.-S. Models for multiple attribute decision-making with intuitionistic fuzzy information. Int. J. Uncertain . Fuzziness Knowl.-Based Syst. 2007, 15, 285-297. [CrossRef]

6. Jayagowri, P.; Geetharamani, G. A Critical Path Problem Using Intuitionistic Trapezoidal Fuzzy Number. Appl. Math. Sci. 2014, 8, 2555-2562.

7. Wan, S.P.; Dong, J.Y. Method of intuitionistic trapezoidal fuzzy number for multi-attribute group decision. Control Decis. 2010, 25, 773-776.

8. Wang, J.Q. Multi-Criteria Interval Intuitionistic Fuzzy Decision-making Approach with Incomplete Certain Information. Control Decis. 2006, 21, 1253-1256, 1263.

9. Wei, G.W. A Method of Interval-Valued Intuitionistic Fuzzy Multiple Attributes Decision Making with Incomplete Attribute Weight Information. Chin. J. Manag. 2008, 5, 208-211, 217.

10. Wan, S.P. Method of attitude index for interval multi-attribute decision-making. Control Decis. 2009, 24, 35-38, 43.

11. Chen, X.H.; Yang, X. Multiple attributive group decision making method based on triangular fuzzy numbers. Syst. Eng. Electron. 2008, 30, 278-282.

12. Wang, J.Q.; Zhang, Z. Aggregation operators on intuitionistic trapezoidal fuzzy number and its application to multi-criteria decision making problems. J. Syst. Eng. Electron. 2009, 20, 321-326.

13. Chen, X.H.; Hu, W.H.; Cao, Y. Hierarchical Multiple Objective Programming Model for Multiple Attribute Decision Making Problems Based on Trapezoidal Fuzzy Numbers. J. Ind. Eng. Eng. Manag. 2012, 26, 192-198.

14. Chen, X. Multiple attributes decision-making model based on exponential type fuzzy numbers and its application. Comput. Eng. Appl. 2013, 49, 116-118, 132.

15. Xu, Z.S.; Wang, H. Managing multi-granularity linguistic information in qualitative group decision making: An overview. Granul. Comput. 2016, 1, 21-35. [CrossRef] 
16. Yang, H.Y.; Lin, L.Y.; Yu, Z.H. A class of fuzzy multiple attributes topsis decision making based on exponential type fuzzy numbers. Comput. Eng. Appl. 2012, 48, 120-124.

17. Gu, C.L.; Zhu, S.F. Multiple Attributes Decision-making Based on Exponential Type Fuzzy Numbers. Fuzzy Syst. Math. 2015, 29, 167-173.

18. Mendel, J.M. A comparison of three approaches for estimating (synthesizing) an interval type-2 fuzzy set model of a linguistic term for computing with words. Granul. Comput. 2016, 1, 59-69. [CrossRef]

(C) 2016 by the authors; licensee MDPI, Basel, Switzerland. This article is an open access article distributed under the terms and conditions of the Creative Commons Attribution (CC-BY) license (http://creativecommons.org/licenses/by/4.0/). 